\title{
Evaluación del tanque Imhoff en el tratamiento de las aguas residuales en el Municipio de Colmenar, Málaga
}

\author{
Evaluation of Imhoff tank in the treatment of wastewater in the Municipality \\ of Colmenar, Malaga \\ Vladimir León Alfonso Menacho ${ }^{1}$
}

\section{RESUMEN}

La depuración de aguas residuales en la depuradora de Colmenar se realiza mediante el uso de biodiscos y del sistema denominado tanque Imhoff, siendo está gestionada por el Consorcio Provincial del Agua de Málaga. El presente trabajo evalua la capacidad depuradora del sistema de tanque Imhoff, basándose en el análisis de parámetros fisicoquímicos en diferentes puntos del sistema a lo largo de un período de tiempo determinado. Esta evaluación se basa en el estudio de la caracterización macroscópica de los fangos del sistema. Se concluye que, si bien la depuradora cumple con los parámetros de vertidos, esto no ocurre todos los meses debido a la elevada carga contaminante en la entrada de la EDAR. El funcionamiento del tanque Imhoff es deficiente, el decantador primario presenta rendimiento cero y negativo de la $\mathrm{DBO}_{5}$ y los sólidos en suspensión, el digestor en relación al pH, sólidos suspendidos, potencial redox, el contenido de materia orgánica y la DQO no presentan las características idóneas para su buen funcionamiento en la mayoría de meses. La caracterización macroscópica de los fangos en las probetas depende principalmente del contenido de sólidos en suspensión y de los gases, esto hace que el fango y los gases se desplacen.

Palabras clave: tanque Imhoff; EDAR; decantador primario; digestor; caracterización macroscópica.

\begin{abstract}
Purifying wastewater in the Colmenar treatment plant is performed by using the system called biodiscs and Imhoff tank, it is being managed by the Provincial Water Consortium of Malaga. This research focuses on the purification evaluation capacity of the Imhoff tank system, based on the analysis of physical-chemical parameters in
\end{abstract}

1 Universidad de Málaga. Málaga, España. 
different parts of the system throughout a period of time. Furthermore this evaluation will be based on the study of macroscopic characterization of sludge of the system. It is concluded that while the treatment plant complies with parameters of discharges, it does not happen every month due to the high pollutant load in the inlet of the EDAR. Operation Imhoff tank is poor, the primary decanter has zero and negative performance of $\mathrm{DBO}_{5}$ and suspended solids, the digester according to the parameters of $\mathrm{pH}$, suspended solids, redox potential, organic matter content and DQO not It presents the ideal characteristics for good performance in most months. Macroscopic characterization of sludges depend mainly on the content of suspended solids and gases, these make the sludge and gases displace into the test tubes.

Keywords: Imhoff tank; EDAR; primary decanter; digester; macroscopic characterization.

\section{ICHIKLLACHAW}

Quchaqashqa yakukunapa tsuyantsayninqa Kulminar nishqan tsuyantsaychawmi rurakan, kayqa biyudiskuswan Imhoff nishqan kitawanmi rurakan, kay kananpaqqa Málagapa Suyum yuritsimurqan. Kay musyakuyqa alli tsuyantsaynin Imhoff kitanpa chanintsaynintam pushan. Kayqa paramitrukuna hwisiku-kimikukunatam analisan, tukuy chikukunachaw huk patsa riqishqa churakashqallachaw. Hinaman kay musyapakuyqa liqitakunapa makruskupikapa chanintsayninkunam. Uchuk shimillachaw tsayta nintsik: kay tsuyantsay paramitruwan tinkuptinqa, chaptinqa, manam llapan killakunatsu tinkun, imanir EDARpa yaykunanchaw allaapa rakchatay kanqanraykur. Imhoff kitan kuyuyninqa mana allitsu, punta kaq dikantadur hutishqa chusyaqmanmi chan, hina DBOpis mana allimanmi chan, llapan chukru kaqkunapis $\mathrm{pH}$ hutishqapis dihisturpis qipatsaariyanmi, hinaman DQOpis manam llapan killakunachaw alliqllaqa kuyukuntsu. Liqitakunapa makruskupikapa kayninqa prubitachaw llapan chukru chawayninmami chan, hinaman wayra kayninpis chanmi, tsayraykurmi liqitawan wayraqa mana nakashpa aywayan.

Pushaq shimikuna: Imhoff hutishqa kita; EDAR nishqan, punta kaq dikantadur, dihistur, makruskupiku imanaw kanqan.

\section{INTRODUCCIÓN}

Las aguas residuales urbanas se definen a las aguas residuales de origen doméstico, o la mezcla de éstas con aguas de origen residual industrial o con aguas de escorrentía pluvial (Ministerio de Obras Públicas, 1995). Para ello, se emplean diferentes sistemas depuradores para pequeñas y grandes aglomeraciones que se le denominarán EDAR (Estación depuradora de aguas residuales). Las instalaciones de tratamiento de aguas residuales, constan de 3 elementos básicos: La recogida y conducción, el tratamiento y la evacuación (CENTA, 2008). En el tratamiento se encuentra el tanque Imhoff que cumple la función de digestor y decantador. 
El tanque Imhoff se define como un sistema de tratamiento primario para poblaciones tributarias menores a 5000 hab, tiene por finalidad remover sólidos en suspensión (OPS, 2005). El proceso consiste en una sedimentación de los sólidos, seguida de una digestión anaerobia de la materia orgánica y de los lodos generados (Blancas y otros, 1994). El tanque Imhoff consta de 3 componentes (figura 1), que son la zona de ventilación, la zona de decantación y la zona de digestión.

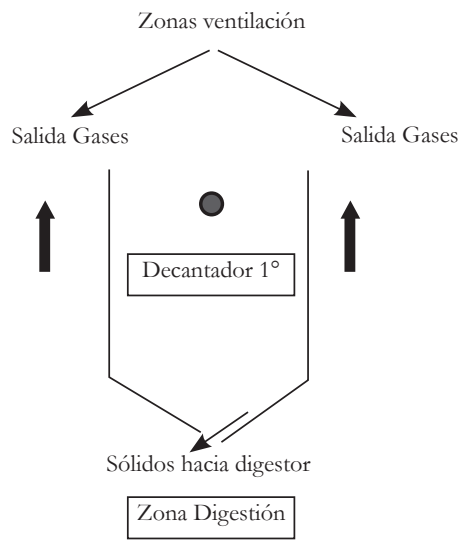

Figura 1. Componentes del tanque Imhoff

El tanque Imhoff en relación a su dimensionamiento separa dos zonas, la zona de decantación se dimensiona como correspondería a un decantador primario y la zona de digestión a un digestor (Riera, 2011). El decantador primario tiene por función la eliminación por la acción prolongada de la gravedad de los sólidos en suspensión más fácilmente sedimentables (Ramalho, 1996). El digestor consiste en un proceso de sedimentación de los sólidos, seguida por la digestión anaerobia de la materia orgánica y de los lodos generados.

La caracterización macroscópica de los fangos hace referencia al ensayo conocido de la V30, se utilizan probetas de graduación de $10 \mathrm{ml}$ o de $20 \mathrm{ml}$ con capacidad de $1000 \mathrm{ml}$ (Zornoza, 2007). El conjunto de características microscópicas del fango que se recogen, se refieren al examen del estado morfológico y estructural del floculo de fango. La evaluación de estas características proporciona información sobre las propiedades de decantación y compactación del fango mezclado (Bello, 2013).

La legislación para el tratamiento de las aguas residuales para cuerpos receptores en Europa está regulada por la Comunidad Económica Europea (CEE), en España por el Ministerio de Agricultura, Alimentación y Medio Ambiente, y a nivel local por los municipios a través de las ordenanzas municipales de vertidos de las aguas residuales a la red de alcantarillado. La normativa europea regula a través de la Directiva No 91/271/ CEE y estado español a través del Real Decreto 509/1996, cabe mencionar que la di- 
rectiva española viene a ser en sí, la adaptación de la normativa europea y el municipio de Colmenar aplica la ordenanza Municipal de Vertidos para las aguas residuales a la red de alcantarillado antes de ser tratadas en la EDAR (Ministerio de Obras Públicas , 1996).

El presente trabajo tiene como objetivos la evaluación de las aguas residuales en la entrada y salida de la EDAR, la evaluación del funcionamiento del tanque Imhoff y la caracterización macroscópica de los fangos en el tanque Imhoff.

\section{MATERIALES Y MÉTODOS}

La evaluación del rendimiento de la EDAR y el decantador primario se hace comparando los valores mensuales de las analíticas en la entrada y salida de los sistemas. El rendimiento de la EDAR se compara con la Directiva $N^{\circ}$ 91/271/CEE, ya sea la concentración o la reducción del contaminante (rendimiento, $(\%)$ ) y en el caso del decantador primario se comparan con los valores del pliego de prescripción técnica de la EDAR. Para determinar el rendimiento se aplica la siguiente fórmula:

$$
\text { Rendimiento }(\%)=\frac{\text { Concentración de entrada }- \text { Concentración de salida }}{\text { Concentración de entrada }} \times 100
$$

La evaluación del digestor del tanque Imhoff se realizó a 2.2 m, 4.0 m y 5.0 m del mismo. La descripción de los parámetros que se evalúan en la EDAR y en el tanque Imhoff (decantador primario y digestor) se representan a continuación:

Tabla 1. Parámetros utilizados en la evaluación de la EDAR y el tanque Imhoff

\begin{tabular}{ccccccc}
\hline & \multicolumn{7}{c}{ Descriptores } \\
\hline & $\mathrm{DBO}_{5}$ & DQO & SS & SSV & pH & PR \\
Entrada/Salida EDAR & $\checkmark$ & $\checkmark$ & $\checkmark$ & & & \\
Decantador primario & $\checkmark$ & & $\checkmark$ & & & \\
Digestor & & $\checkmark$ & $\checkmark$ & $\checkmark$ & $\checkmark$ & $\checkmark$ \\
\hline
\end{tabular}

* DBO5: Demandad bioquímica de oxígeno

* DQO: Demanda química de oxígeno

* SSV: Sólidos suspendidos volátiles

* SS: Sólidos en suspensión

* PR: Potencial redox 
Los informes de laboratorio con las variables evaluadas en la EDAR y del tanque Imhoff (decantador primario y digestor) se recopila de manera mensual entre los meses de noviembre del 2015 a mayo del 2016 dada la facilidad del Consorcio Provincial del Agua Málaga y del Laboratorio de Ensayo y Calibración D.NOTA.

La caracterización macroscópica de los fangos utiliza los fangos procedentes del decantador secundario al Imhoff, el fango que llega del fondo del Imhoff al espesador y el fango que a distintas alturas en el interior del Imhoff ( $4.0 \mathrm{~m}$ y $5.0 \mathrm{~m}$ ). Para ello, se recoge $1000 \mathrm{ml}$ de fangos en probetas de la misma capacidad. En la tabla 2 se describen las consideraciones:

Tabla 2. Consideraciones para el ensayo del fango de diferentes procedencias en el tanque Imhoff

\begin{tabular}{ccccc}
\hline Procedencia del fango & $\begin{array}{c}\text { Tipo de } \\
\text { probeta }\end{array}$ & $\begin{array}{c}\text { Numero de } \\
\text { probetas }\end{array}$ & $\begin{array}{c}\text { Frecuencia de } \\
\text { medición (h) }\end{array}$ & $\begin{array}{c}\text { Duración de } \\
\text { incubación }\end{array}$ \\
\hline $\begin{array}{c}\text { Fango del decantador } \\
\text { secundario, réplica 1 }\end{array}$ & Boca ancha & 3 & $0,5-1$ & $4 \mathrm{~h}$ \\
$\begin{array}{c}\text { Fango del decantador } \\
\text { secundario, réplica 2 }\end{array}$ & Boca corta & 3 & $1-2,5$ & $6,5 \mathrm{~h}$ \\
Fango del espesador & Boca ancha & 3 & $0,5-2$ & $7 \mathrm{~h}$ \\
Fango a 4.0m & Boca corta & 3 & $2-67$ & $260 \mathrm{~h}$ \\
Fango a 5.0m & Boca ancha & 3 & $2-67$ & $260 \mathrm{~h}$ \\
\hline
\end{tabular}

La descripción del proceso de decantación consiste en registrar los valores de la decantabilidad del fango en la probeta de $1000 \mathrm{ml}$ a lo largo del tiempo de acuerdo a las frecuencias de medición detalladas. También se registran las observaciones de las características del clarificado del fango, siendo las características observadas del olor, color, presencia de espumas, grasas o emulsiones, aspecto de decantación, la presencia de fisuras, los sólidos en el clarificado y la turbidez (RAITEC, 2009). Estos ensayos se realizan a temperatura ambiental.

No se aplican métodos estadísticos en la evaluación de la EDAR, el tanque Imohff (decantador primario y digestor) y en la caracterización macroscópica de los fangos debido a que en los informes de las variables evaluadas reportan un solo valor, mas no repeticiones y en caso de la caracterización macroscópica de los fangos se presentan el comportamiento y las características del fango en las probetas a diferentes horas. 


\section{RESULTADOS}

Los informes de las analíticas para la EDAR y el tanque Imhoff se presentan a continuación:

Tabla 3. Valores de las variables en la entrada y salida de la EDAR y el decantador primario (DBO5 (mg/l); DQO (mg/l); SS (mg/l)) con el tiempo (meses)

\begin{tabular}{|c|c|c|c|c|c|c|c|c|c|c|}
\hline \multirow{3}{*}{ Mes } & \multicolumn{6}{|c|}{ EDAR } & \multicolumn{4}{|c|}{ Decantador primario } \\
\hline & \multicolumn{3}{|c|}{ Entrada } & \multicolumn{3}{|c|}{ Salida } & \multicolumn{2}{|c|}{ Entrada } & \multicolumn{2}{|c|}{ Salida } \\
\hline & $\mathrm{DBO}_{5}$ & DQO & SS & $\mathrm{DBO}_{5}$ & DQO & SS & $\mathrm{DBO}_{5}$ & SS & $\mathrm{DBO}_{5}$ & SS \\
\hline $\begin{array}{c}\text { No- } \\
\text { viembre }\end{array}$ & 59 & 166 & 66 & 22 & 71 & 29,2 & 121 & 549 & 30 & 89 \\
\hline $\begin{array}{c}\text { Diciem- } \\
\text { bre }\end{array}$ & 1818 & 4901 & 3345 & 331 & 961 & 50 & 462 & 720 & 461 & 1007 \\
\hline Enero & 67 & 240 & 164 & 12 & 29 & 21 & 352 & 560 & 458 & 1100 \\
\hline Febrero & 175 & 505 & 168 & 31 & 104 & 34 & 263 & 386 & 196 & 172 \\
\hline Marzo & 127 & 345 & 114 & 18 & 48 & 18 & 84 & 139 & 120 & 186 \\
\hline Abril & 80 & 250 & 105 & 11 & 38 & 5,2 & 47 & 224 & 42 & 178 \\
\hline Mayo & 30 & 100 & 73 & 22 & 98 & 61 & 53 & 76 & 57 & 146 \\
\hline
\end{tabular}

Fuente: Laboratorio D.NOTA

Tabla 4. Valores de las variables en el tanque Imhoff a $2.2 \mathrm{~m}, 4.0 \mathrm{~m}$ y $5.0 \mathrm{~m}$ (pH; SS (mg/l); $\mathrm{SSV}(\mathrm{mg} / \mathrm{l}) ; \mathrm{PR}(\mathrm{mv}) ; \mathrm{DQO}(\mathrm{mg} / \mathrm{l})$ ) con el tiempo (meses)

\begin{tabular}{ccccccccccccccc}
\hline Mes & \multicolumn{4}{c}{ Imhoff a $2.2 \mathrm{~m}$} & \multicolumn{1}{c}{ Imhoff a $4.0 \mathrm{~m}$} & \multicolumn{1}{c}{ Imhoff a 5.0m } \\
& $\mathrm{pH}$ & $\mathrm{SS}$ & SSV & PR & pH & SS & SSV & PR & DQO & pH & SS & SSV & PR & DQO \\
\hline $\begin{array}{c}\text { No- } \\
\text { viembre }\end{array}$ & 7,3 & 424 & 292 & -85 & 6,7 & 27600 & 16660 & 19,9 & 46600 & 6,8 & 28360 & 17060 & -111 & NE \\
$\begin{array}{c}\text { Diciem- } \\
\text { bre }\end{array}$ & 7,2 & 4420 & 3320 & -269 & 6,5 & 29800 & 19980 & -127 & 19031 & 6,4 & 41467 & 27833 & -176 & NE \\
Enero & 7,2 & 485 & 440 & -274 & 6,5 & 54450 & 39150 & -84 & 27778 & 6,4 & 44300 & 31850 & -76 & NE \\
Febrero & 8,2 & 282 & 234 & -252 & 6,2 & 37420 & 27080 & -135 & 50000 & 8,2 & 40460 & 29000 & -106 & NE \\
Marzo & 7,6 & 162 & 144 & -249 & 7,5 & 135 & 120 & -240 & 450 & 6,5 & 35800 & 26467 & -134 & 46500 \\
Abril & 7,5 & 170 & 160 & -234 & 7,5 & 164 & 168 & -219 & 300 & 6,6 & 33600 & 27200 & -166 & 93000 \\
Mayo & 7,9 & 142 & 122 & NE & 6,9 & 36600 & 24250 & NE & 75000 & 7,2 & 34144 & 22567 & NE & 37500 \\
\hline
\end{tabular}

Fuente: Laboratorio D.NOTA

Los resultados de la evaluación del rendimiento/concentración de la EDAR se presentan a continuación: 
Tabla 5. $\mathrm{DBO}_{5}(\mathrm{mg} / \mathrm{l})$ a la entrada y salida de la EDAR, rendimiento (\%) y cumplimiento de la Directiva $\mathrm{N}^{\circ} 91 / 271 / \mathrm{CEE}(25 \mathrm{mg} / 1$ o $70-90 \%$ )

\begin{tabular}{cccccc}
\hline Mes & $\begin{array}{c}\text { Entrada } \\
\mathrm{DBO}_{5}\end{array}$ & Salida $\mathrm{DBO}_{5}$ & $\begin{array}{c}\text { Rendimiento } \\
\mathrm{DBO}_{5} \\
\mathrm{DBO}_{5}(25 \mathrm{mg} / \mathrm{l})\end{array}$ & $\begin{array}{c}\mathrm{DBO}_{5} \\
(70-90 \%)\end{array}$ & \\
\hline Noviembre & 59 & 22 & $62,7 \%$ & Sí & NO \\
Diciembre & 1818 & 331 & $81,8 \%$ & NO & SÍ \\
Enero & 67 & 12 & $82,1 \%$ & SI & Sí \\
Febrero & 175 & 31 & $82,3 \%$ & NO & Sí \\
Marzo & 127 & 18 & $85,8 \%$ & Sí & Sí \\
Abril & 80 & 11 & $86,3 \%$ & Sí & Sí \\
Mayo & 30 & 22 & $26,7 \%$ & Sí & NO \\
Promedio & $89,7^{*}$ & $19,3^{*}$ & & & \\
\hline
\end{tabular}

89, $7^{*}$ : Promedio entrada de la $\mathrm{DBO}_{5}$ sin considerar el valor de diciembre. 19,3*: Promedio salida de la $\mathrm{DBO}_{5}$ sin considerar el valor de diciembre.

Tabla 6. DQO (mg/l) a la entrada y salida de la EDAR, rendimiento (\%) y cumplimiento de la Directiva N$^{\circ}$ 91/271/CEE (125mg/1 o 75\%)

\begin{tabular}{cccccc}
\hline Mes & $\begin{array}{c}\text { Entrada } \\
\text { DQO }\end{array}$ & Salida & $\begin{array}{c}\text { Rendimiento } \\
\text { DQO }\end{array}$ & $\begin{array}{c}\text { Cumplimiento } \\
\text { DQO } \\
(125 \mathrm{mg} / \mathrm{l})\end{array}$ & DQO $(75 \%)$ \\
\hline Noviembre & 166 & 71 & $57,2 \%$ & SÍ & NO \\
Diciembre & 4901 & 961 & $80,4 \%$ & NO & SÍ \\
Enero & 240 & 29 & $87,9 \%$ & SÍ & SÍ \\
Febrero & 505 & 104 & $79,4 \%$ & SÍ & SÍ \\
Marzo & 345 & 48 & $86,1 \%$ & Sí & Sí \\
Abril & 250 & 38 & $84,8 \%$ & SÍ & Sí \\
Mayo & 100 & 98 & $2,0 \%$ & SÍ & NO \\
Promedio & $267,7^{*}$ & $64,7^{*}$ & & & \\
\hline
\end{tabular}

267, $7^{*}$ : Promedio entrada de la DQO sin considerar el valor de diciembre 64,7*: Promedio salida de la DQO sin considerar el valor de diciembre 
Tabla 7. SS (mg/l) a la entrada y salida de la EDAR, rendimiento (\%) y cumplimiento de la Directiva No 91/271/CEE (35mg/l o 90\%)

\begin{tabular}{cccccc}
\hline Mes & $\begin{array}{c}\text { Entrada } \\
\text { SS }\end{array}$ & Salida SS & $\begin{array}{c}\text { Rendimiento } \\
\text { SS }\end{array}$ & \multicolumn{2}{c}{ Cumplimiento } \\
SS (35mg/l) & SS (90\%) \\
\hline Noviembre & 66 & 29,2 & $55,8 \%$ & SÍ & NO \\
Diciembre & 3345 & 50 & $98,5 \%$ & NO & SÍ \\
Enero & 164 & 21 & $87,2 \%$ & Sí & NO \\
Febrero & 168 & 34 & $79,8 \%$ & Sí & NO \\
Marzo & 114 & 18 & $84,2 \%$ & Sí & NO \\
Abril & 105 & 5,2 & $95,1 \%$ & SÍ & SÍ \\
Mayo & 73 & 61 & $16,4 \%$ & NO & NO \\
Promedio & $115^{*}$ & $28,1^{*}$ & & & \\
\hline
\end{tabular}

115*: Promedio entrada de los SS sin considerar el valor de diciembre 28,1*: Promedio salida de los SS sin considerar el valor de diciembre

Los resultados de la evaluación del rendimiento del decantador primario se presentan a continuación:

Tabla 8. $\mathrm{DBO}_{5}(\mathrm{mg} / \mathrm{l})$ a la entrada y salida del decantador primario, rendimiento (\%) y cumplimento del pliego de prescripción técnica de la EDAR (30\%)

\begin{tabular}{ccccc}
\hline Mes & $\begin{array}{c}\text { Entrada } \\
\mathrm{DBO}_{5}\end{array}$ & $\begin{array}{c}\text { Salida } \\
\mathrm{DBO}_{5}\end{array}$ & $\begin{array}{c}\text { Rendimiento } \\
\mathrm{DBO}_{5}\end{array}$ & $\begin{array}{c}\text { Cumplimiento } \\
\mathrm{DBO}_{5}(30 \%)\end{array}$ \\
\hline Noviembre & 121 & 30 & $75,2 \%$ & SÍ \\
Diciembre & 462 & 461 & $0,2 \%$ & $\mathrm{NO}$ \\
Enero & 352 & 458 & $-30,1 \%$ & $\mathrm{NO}$ \\
Febrero & 263 & 196 & $25,5 \%$ & $\mathrm{NO}$ \\
Marzo & 84 & 120 & $-42,9 \%$ & $\mathrm{NO}$ \\
Abril & 47 & 42 & $10,6 \%$ & $\mathrm{NO}$ \\
Mayo & 53 & 57 & $-7,5 \%$ & $\mathrm{NO}$ \\
Promedio & 197,4 & 194,9 & $0,0 \%$ & \\
\hline
\end{tabular}

Tabla 9. SS (mg/l) a la entrada y salida del decantador primario, rendimiento (\%) y cumplimento del pliego de prescripción técnica de la EDAR (60\%)

\begin{tabular}{ccccc}
\hline Mes & Entrada SS & $\begin{array}{c}\text { Salida } \\
\text { SS }\end{array}$ & $\begin{array}{c}\text { Rendimiento } \\
\text { SS }\end{array}$ & $\begin{array}{c}\text { Cumplimiento } \\
\text { SS }(60 \%)\end{array}$ \\
\hline Noviembre & 549 & 89 & $83,8 \%$ & SÍ \\
Diciembre & 720 & 1007 & $-39,9 \%$ & NO \\
Enero & 560 & 1100 & $-96,4 \%$ & NO \\
Febrero & 386 & 172 & $55,4 \%$ & NO \\
Marzo & 139 & 186 & $-33,8 \%$ & NO \\
Abril & 224 & 178 & $20,5 \%$ & NO \\
Mayo & 76 & 146 & $-92,1 \%$ & NO \\
Promedio & 379,1 & 411,1 & $-0,1 \%$ & \\
\hline
\end{tabular}


Los resultados de la evaluación de la zona de digestión se presentan a continuación:

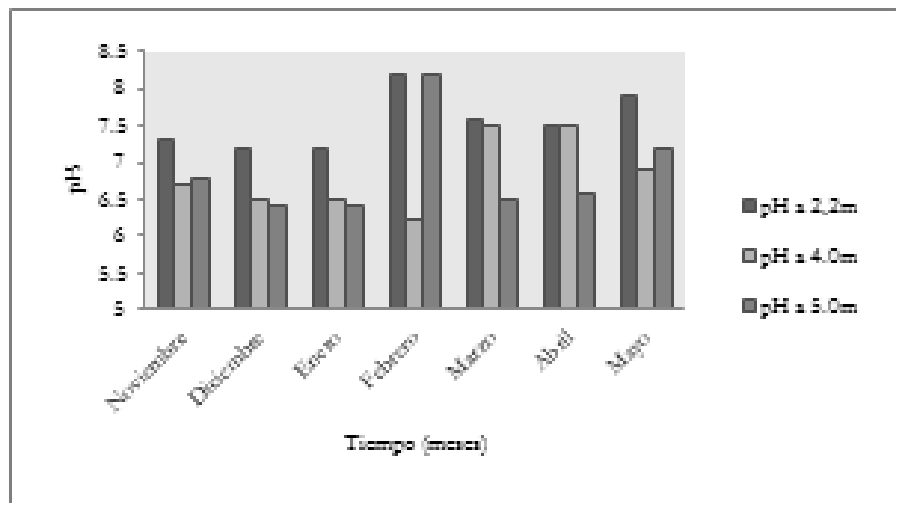

Figura 2. $\mathrm{pH}$ a diferentes alturas $(2.2 \mathrm{~m}, 4.0 \mathrm{~m}, 5.0 \mathrm{~m})$ en el interior del tanque Imhoff a lo largo del tiempo (meses)

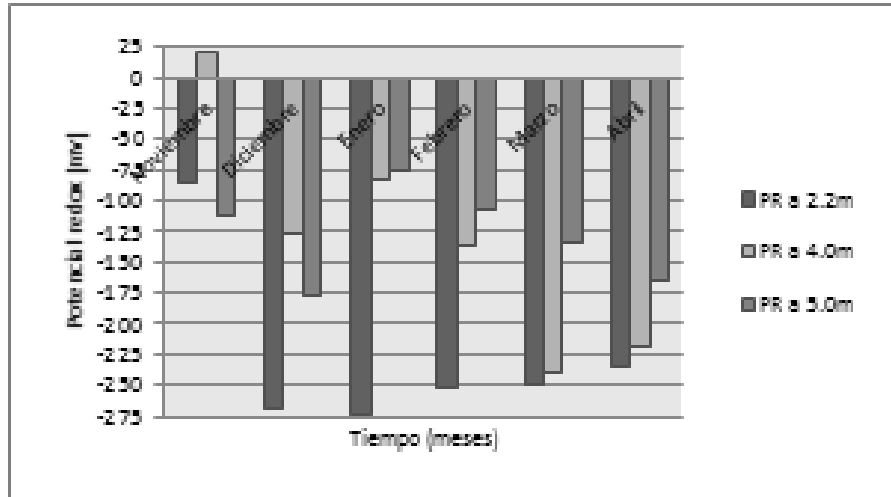

Figura 3. (SS) (g/l) a diferentes alturas $(2.2 \mathrm{~m}, 4.0 \mathrm{~m}, 5.0 \mathrm{~m})$ al interior del tanque Imhoff a lo largo del tiempo (meses)

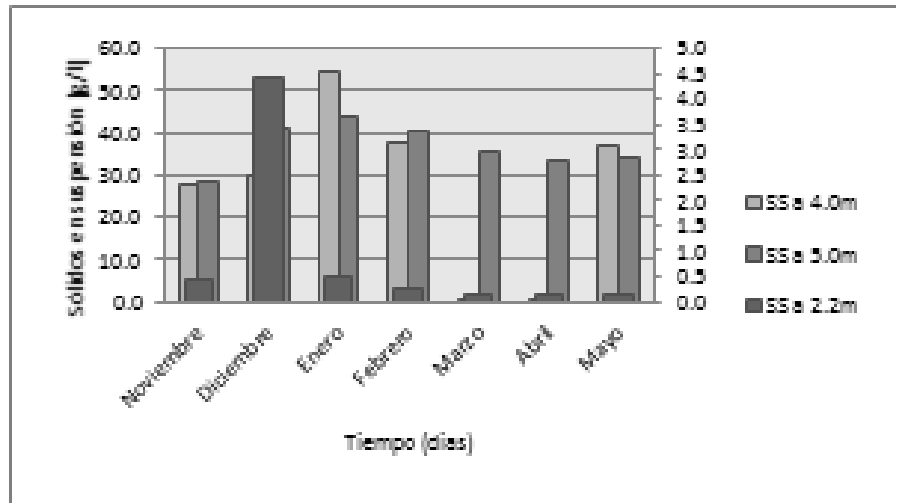

Figura 4. PR (mv) a diferentes alturas $(2.2 \mathrm{~m}, 4.0 \mathrm{~m}, 5.0 \mathrm{~m})$ al interior del tanque Imhoff a lo largo del tiempo (meses) 
Tabla 10. SSV/SS (\%) respecto a las alturas de $2.2 \mathrm{~m}, 4.0 \mathrm{~m}$ y $5.0 \mathrm{~m}$ al interior del tanque Imhoff a lo largo del tiempo (meses)

\begin{tabular}{cccc}
\hline Mes & SSV a 2.2m & SS a 4.0m & SS a 5.0m \\
\hline Noviembre & $68,9 \%$ & $60,4 \%$ & $60,2 \%$ \\
Diciembre & $75,1 \%$ & $67,0 \%$ & $67,1 \%$ \\
Enero & $90,7 \%$ & $71,9 \%$ & $71,9 \%$ \\
Febrero & $83,0 \%$ & $72,4 \%$ & $71,7 \%$ \\
Marzo & $88,9 \%$ & $88,9 \%$ & $73,9 \%$ \\
Abril & $94,1 \%$ & $102,4 \%$ & $81,0 \%$ \\
Mayo & $85,9 \%$ & $66,3 \%$ & $66,1 \%$ \\
Promedio & $83,8 \%$ & $71,1 \% *$ & $70,3 \%$ \\
\hline
\end{tabular}

$71,1^{*}$ : Promedio de la relación SSV/SS (\%) a $4.0 \mathrm{~m}$ sin considerar el valor de abril

Tabla 11. DQO (mg/l) a las alturas de $2.2 \mathrm{~m}, 4.0 \mathrm{~m}$ y $5.0 \mathrm{~m}$ al interior del tanque Imhoff a lo largo del tiempo (meses)

\begin{tabular}{ccc}
\hline Mes & DQO a 4.0m & DQO a 5.0m \\
\hline Noviembre & 46,6 & NE \\
Diciembre & 19,0 & NE \\
Enero & 27,8 & NE \\
Febrero & 50,0 & NE \\
Marzo & 0,5 & 46,5 \\
Abril & 0,3 & 93,0 \\
Mayo & 75,0 & 37,5 \\
Promedio & 31,3 & 59,0 \\
\hline
\end{tabular}

NE: Valor de la DQO no obtenido

Los resultados de la caracterización macroscópica de los fangos que llegan del decantador secundario al Imhoff, el fango que llega del fondo del Imhoff al espesador y el fango que a distintas alturas en el interior del Imhoff $(4.0 \mathrm{~m}$ y $5.0 \mathrm{~m})$ se presentan a continuación: 


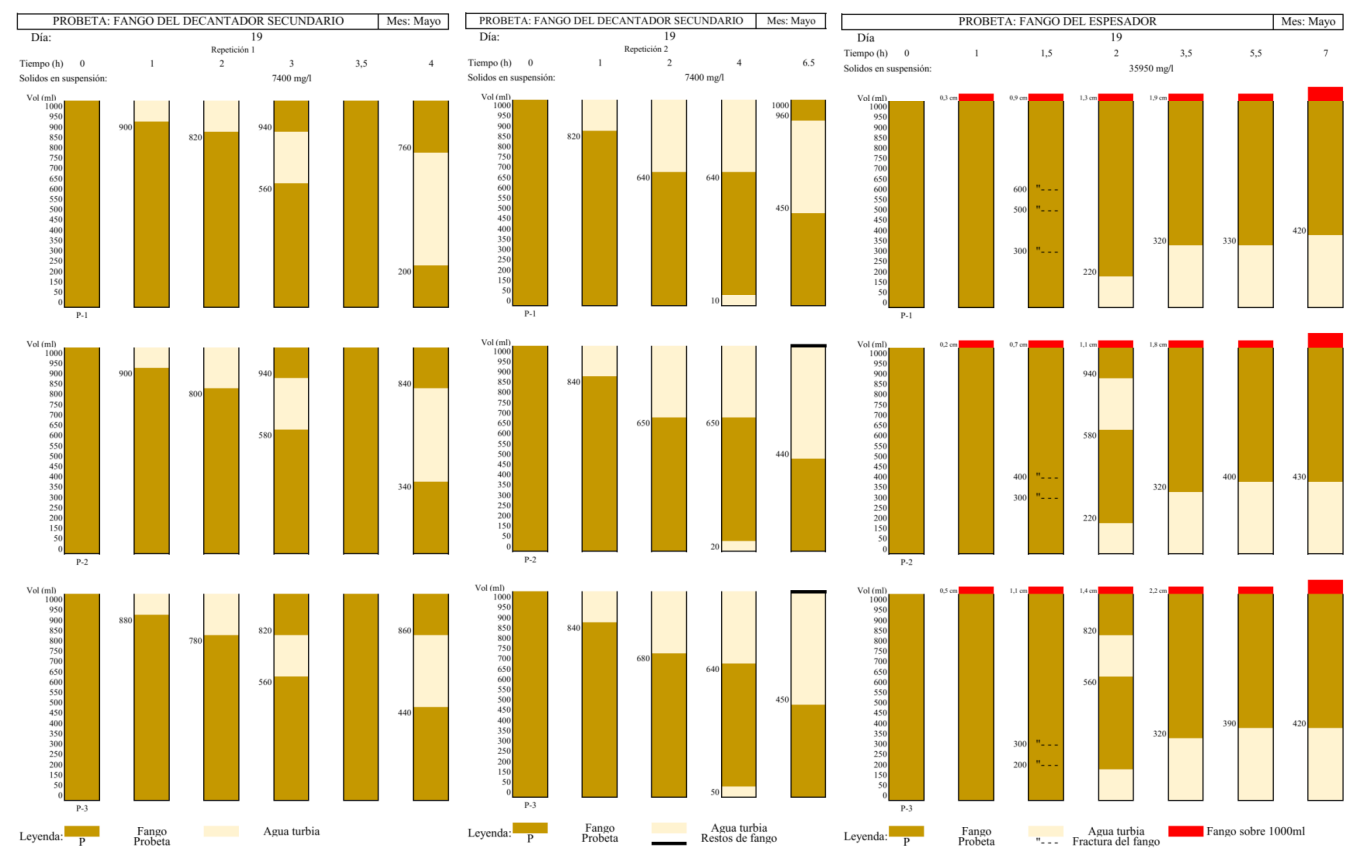

Figura 5. Probetas con fango procedente del decantador secundario repetición 1, repetición 2 y del espesador a lo largo del tiempo (h)

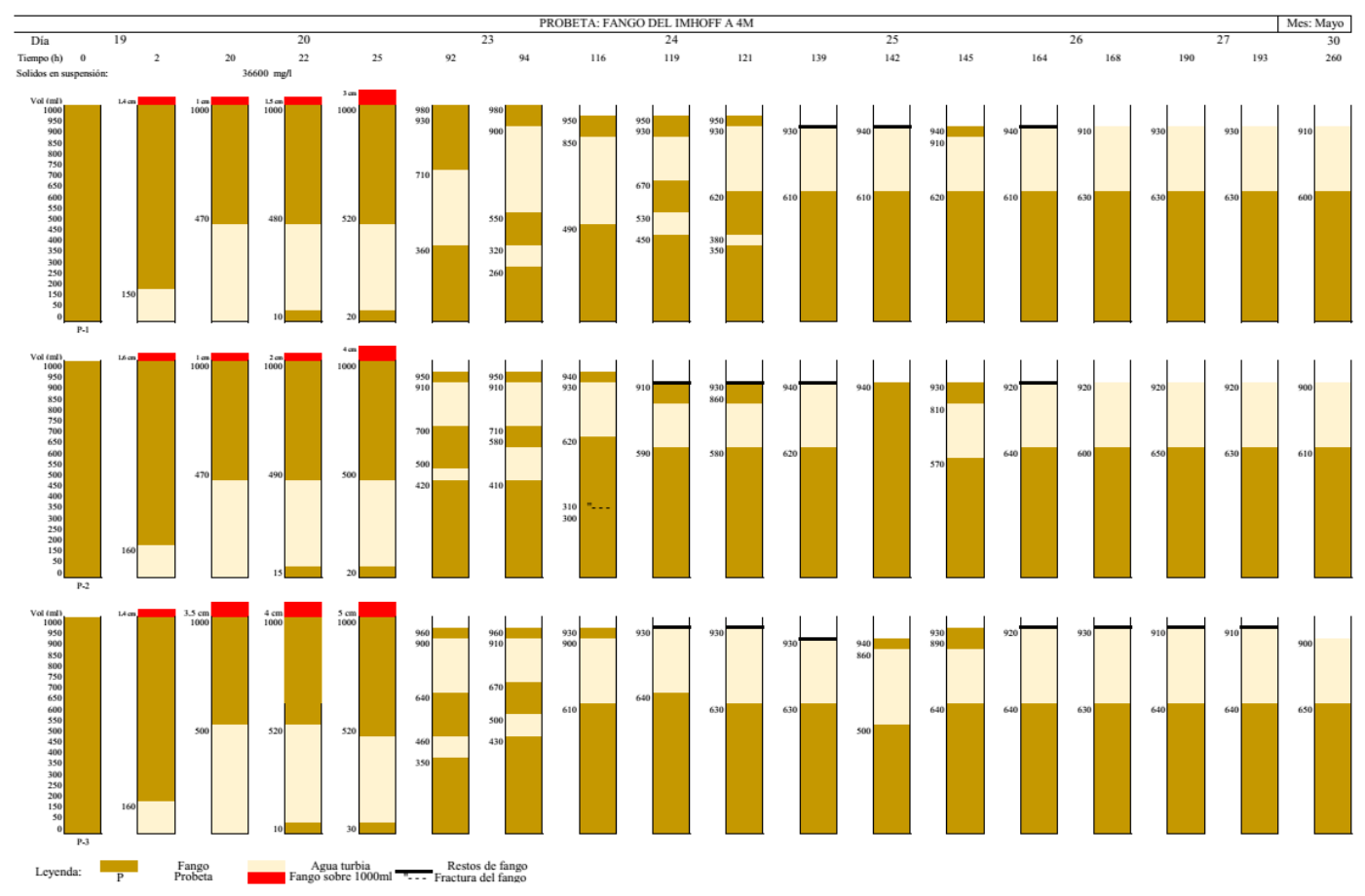

Figura 6. Probetas con fango procedente del tanque Imhoff a $4.0 \mathrm{~m}$ a lo largo del tiempo (h) 


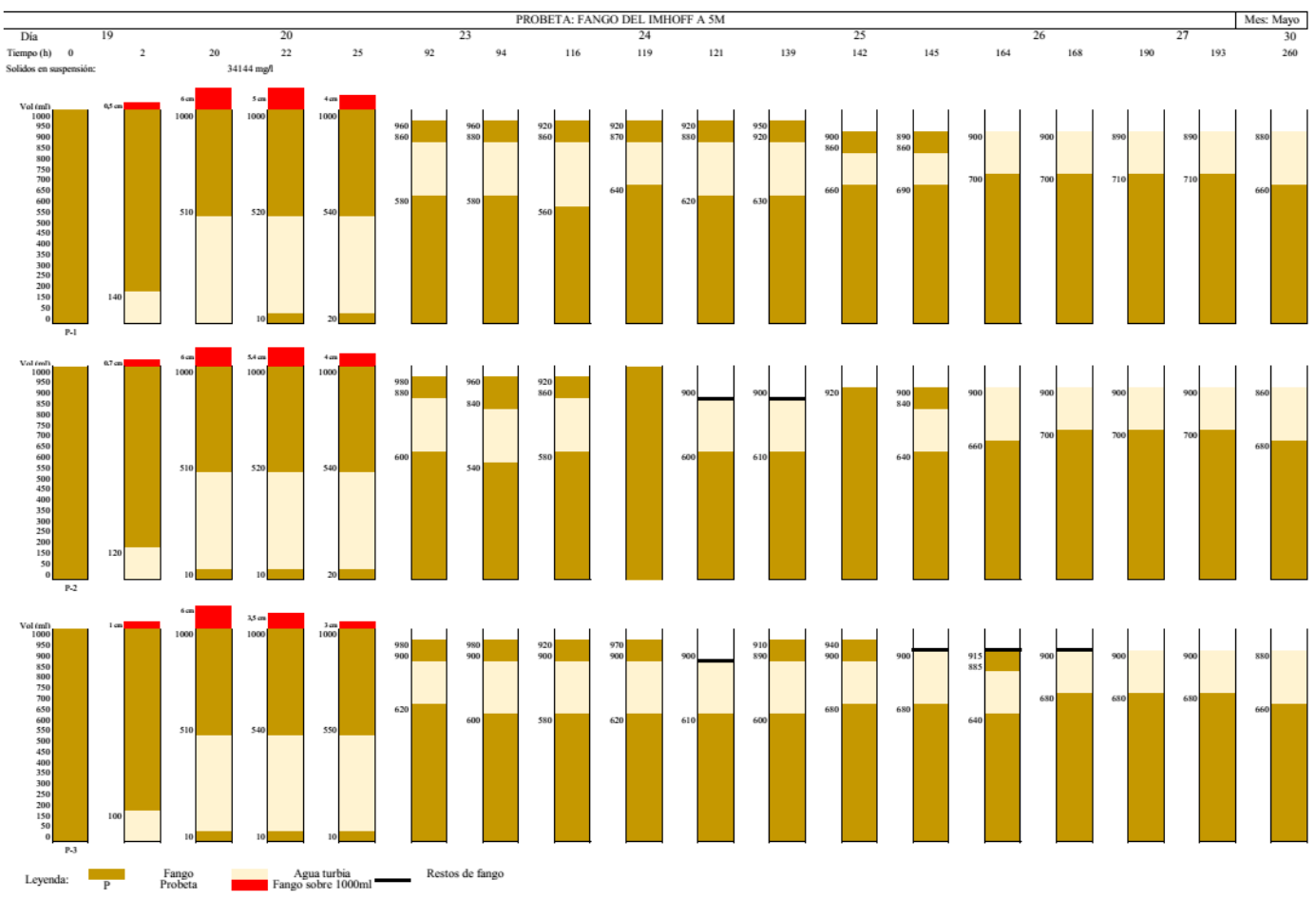

Figura 7. Probetas con fango procedente del tanque Imhoff a 5.0m a lo largo del tiempo (h)

Los resultados del clarificado del fango de diferentes procedencias (tabla 12) muestran las siguientes características:

Tabla 12. Características del clarificado del fango de diferentes procedencias para ensayos con 3 probetas

\begin{tabular}{cccccc}
\hline Características del fango & \multicolumn{2}{c}{ Decantador secundario } & Espesador & $\begin{array}{c}\text { Imhoff a } \\
4.0 \mathrm{~m}\end{array}$ & $\begin{array}{c}\text { Imhoff a } \\
5.0 \mathrm{~m}\end{array}$ \\
\hline Olor & Repetición 1 & Repetición 2 & & Correcto \\
$\begin{array}{c}\text { Espumas, grasas, emulsiones en la } \\
\text { superficie de la probeta }\end{array}$ & Correcto & Correcto & Correcto & Correcto & Sí \\
Aspecto de la decantación & Algodonoso & Algodonoso & En bloque & En bloque & En bloque \\
Fisuras & Sí & Sí & Sí & Sí & Sí \\
Presencia de sólidos en el clarificado & Sí & Sí & Sí & Sí & Sí \\
Turbidez & Muy alta & Muy alta & Muy alta & Muy alta & Muy alta \\
\hline
\end{tabular}




\section{DISCUSIÓN}

La evaluación de la EDAR muestra que en el mes de diciembre la concentración de la $\mathrm{DBO}_{5}$ en la entrada es $1818 \mathrm{mg} / 1$ y en la salida $331 \mathrm{mg} / 1$, estos valores son elevados en comparación con los otros meses, por consiguiente, no se cumple con los límites de vertido $(25 \mathrm{mg} / \mathrm{l})$ pero, si se compara con el rendimiento (70-90\%) se cumple con un valor de $81,79 \%$. La concentración de la DQO en la entrada es $4901 \mathrm{mg} / 1$ y en la salida $961 \mathrm{mg} / \mathrm{l}$, estos valores son elevados en comparación con los otros meses, por consiguiente, no se cumple con los límites de vertido $(125 \mathrm{mg} / \mathrm{l})$ pero, si se compara con el rendimiento $(75 \%)$ se cumple con un valor de 80,4\%. La concentración de los sólidos en suspensión (SS) en la entrada es $3345 \mathrm{mg} / 1$ y en la salida $50 \mathrm{mg} / 1$ estos valores son elevados en comparación con los otros meses, por consiguiente, no se cumple con los límites de vertido $(35 \mathrm{mg} / \mathrm{l})$ pero, si se compara con el rendimiento $(90 \%)$ se cumple con un valor de $98,5 \%$.

La concentración promedio en la entrada y salida de la $\mathrm{DBO}_{5}$, DQO y SS muestran que en todos los meses se reduce la carga contaminante en la EDAR. En diciembre es más que evidente, la elevada carga contaminante en la entrada del sistema, por ello se registran valores elevados y, por consiguiente, su inadecuado funcionamiento.

La evaluación del decantador primario muestra que el rendimiento de la $\mathrm{DBO}_{5}$ no se cumple en todos los meses excepto en noviembre, el valor promedio es $0 \%$, esto quiere decir, que no se cumple la función de decantación en el tanque Imhoff. En comparación con otros autores (Prescripción del pliego del proyecto, el Real Decreto 11/1995, la OPS), tampoco se cumplen los rendimientos esperados. En el caso de los sólidos en suspensión el valor promedio del rendimiento es negativo $(-0.1 \%)$, por tanto, el decantador no cumple la función de reducir el contenido de los sólidos en suspensión.

La deficiencia del decantador primario es el tiempo de retención hidráulica de las aguas residuales en el decantador primario, para el volumen actual de 3,26h (prescripción técnica de la EDAR) no es el adecuado, debería ser mayor para su diseño. Algunos autores consideran otros valores, por ejemplo, la OPS considera de $1,5 \mathrm{~h}$ a $2,5 \mathrm{~h}$ y el RAS de $2 \mathrm{~h}$ a $2,5 \mathrm{~h}$.

La evaluación del digestor muestra que el en el mes de febrero el pH a $2.2 \mathrm{~m}$ y $5.0 \mathrm{~m}$ supera el valor de 8 , esto quiere decir que la digestión del fango es inadecuada porque el $\mathrm{pH}$ debe de estar en el rango de 6 a 8 por pertenecer a un ambiente reductor (Ortega, 2006).

Los sólidos en suspensión incrementan con la profundidad debido a que en la zona de digestión llegan los sólidos decantador primario y del decantador secundario, además se encuentran los fangos digeridos en el proceso anaerobio, por ello, es incorrecto que en los meses enero y mayo la concentración de los sólidos a $4.0 \mathrm{~m}$ sea mayor que a $5.0 \mathrm{~m}$. 
El potencial redox en la digestión anaerobia de los fangos presenta valores negativos que deben mantener por debajo de $-300 \mathrm{mv}$ o $-330 \mathrm{mv}$ (Ortega, 2006) para asegurar un ambiente fuertemente reductor, pero el valor negativo más elevado no sobrepasa los -275mv. Además, los valores negativos altos del potencial redox deben de ser a mayor profundidad, cosa que no se cumple en la mayoría de meses.

El contenido de materia orgánica reducida es mínimo en casi todos los meses a las alturas de $4.0 \mathrm{~m}$ y $5.0 \mathrm{~m}$. La materia orgánica promedio a $4.0 \mathrm{~m}$ es $71,1 \%$ y a $5.0 \mathrm{~m}$ es $70,3 \%$, la reducción es insignificante. Cabe mencionar que a $4.0 \mathrm{~m}$ el valor de la materia orgánica reducida sobrepasa el 100\%, valor que es incorrecto, debido a un dato erróneo reportado por el laboratorio.

Los valores de la DQO deben de incrementase con la profundidad, pero solo ocurre en el mes de abril y no en mayo. El incremento del contenido de la DQO a 5.0m debe ser mayor ya que ahí se encuentran la mayor cantidad de fangos que no se pueden digerir biológicamente, y deben ser mineralizados.

La deficiencia del digestor está relacionada con la temperatura de digestión de lodos y volumen de almacenamiento de los mismos, la OPS considera la temperatura de diseño del digestor a $15^{\circ} \mathrm{C}$, pero las condiciones ambientales no lo permiten. También otro de los posibles defectos es el tiempo de digestión de lodos con la temperatura, en la EDAR en Colmenar, el digestor del tanque Imhoff prevé un tiempo de digestión de 60 días según su diseño, pero comparando con otros autores (Imhoff y OPS), la temperatura promedio a la que debe estar la zona de digestión para esta cantidad de días sería $15^{\circ} \mathrm{C}$, cosa que no se cumple debido a que existen temporadas de invierno y temperaturas muy bajas y poco uniformes.

La caracterización macroscópica de los fangos, muestra al fango que llega del decantador secundario al Imhoff, el fango que llega del fondo del Imhoff al espesador y el fango que a distintas alturas en el interior del Imhoff $(4.0 \mathrm{~m}$ y $5.0 \mathrm{~m})$ muestran diferentes comportamientos debido a la variación de la concentración de los sólidos en suspensión. En el fango del decantador secundario (para ambas repeticiones) la concentración de los sólidos en suspensión es $7400 \mathrm{~m} / 1$, esto permite que los gases y la densidad en relación al desplazamiento del fango sea lenta en la probeta en comparación con los otros fangos. La concentración de los fangos procedentes del espesador, a 4.0m y 5.0m presentan concentraciones de $35950 \mathrm{mg} / 1,36600 \mathrm{mg} / 1,34144 \mathrm{mg} / 1$ respectivamente, son concentraciones elevadas en las probetas, el desplazamiento de fango es muy lenta por el elevado contendido de materia biodegradable, por tanto, el contenido de gases es mayor. También se puede decir que el volumen al final de la probeta será menor que los $1000 \mathrm{ml}$.

Las propiedades del clarificado del fango en las probetas se observaron durante el tiempo de decantación del fango, la limitante es que no existen criterios específicos para evaluar las variables consideradas. 


\section{CONCLUSIONES}

La EDAR en el Municipio de Colmenar, cumple con los rendimientos esperados de la $\mathrm{DBO}_{5}, \mathrm{DQO}$ y Sólidos en suspensión para la mayoría de meses, pero es recomendable aplicar la ordenanza municipal de vertidos para regular la carga contaminante en la entrada de la EDAR y así permitir su buen funcionamiento.

El sistema de Tanque Imhoff es deficiente, debido a que el decantador primario y el digestor presentan rendimientos muy bajos a los esperados, es recomendable implementar otro tipo decantador primario en la EDAR. También se recomienda a investigadores del sistema del tanque Imhoff uniformizar los rendimientos de la $\mathrm{DBO}_{5}$, DQO, SS, tiempo de retención hidráulica, tiempo de digestión de lodos y temperatura de digestión de lodos para hacer una buena evaluación del sistema.

El comportamiento de los fangos del decantador secundario al Imhoff, del fondo del Imhoff al espesador y el fango a $4.0 \mathrm{~m}$ y $5.0 \mathrm{~m}$ en el interior del Imhoff dependen del contenido de sólidos en suspensión y los gases que se generan. El proceso inicia con el llenado del fango en la probeta, el cuál empieza a decantar (aunque no se observa esto en todos los casos), después por efecto de los gases el fango se desplaza a la parte superior de la probeta y el agua desciende en el fondo; luego el fango se distribuye por secciones, en la parte superior de la probeta se ubica el fango, en la parte intermedia agua y en el fondo fango. El fango finalmente decanta, el volumen de fango y agua en la probeta a medida que incrementa el tiempo es menor a la inicial.

Las propiedades del clarificado del fango durante el tiempo de observación no cuentan con una metodología para su evaluación.

\section{AGRADECIMIENTOS}

Agradecimientos a PhD. María Altamirano Jeschke de la Universidad de Málaga y al Sr. José Luis Ríos Aragüez del Consorcio Provincial del Agua de Málaga.

\section{REFERENCIAS BIBLIOGRÁFICAS}

Bello, 2013. «Bioindicación para el control del proceso de tratamiento biológico de aguas residuales industriales en la EDAR de helados Alacant, estudio comparativo entre esta biofauna y la del MBR piloto de Rincón de león». A. T. Juame. Alicante: Trabajo de fin de Master.

Blancas, Carmen y otros. 1994. Criterios para la evaluación sanitaria de proyectos de reutilización directa de aguas residuales urbanas depuradas. Granada: Talleres Gráficos Arte Junerias y Cia. S.L.

CENTA. 2008. «Manual de depuración de aguas residuales urbanas». Sevilla: Ideasamares. 34-38. 
Colmenar, 2013. «Plan general de ordenación urbanística de Colmenar, ordenanzas municipales». Colmenar.

Ministerio de Obras Públicas. 1995. «REAL DECRETO-LEY 11/1995». Boletín Oficial del Estado. 37518.

Ministerio de Obras Públicas. 1996. «REAL DECRETO 509/1996». Boletín oficial.

OPS. 2005. «Guía para el diseño de tanques sépticos, tanques Imhoff y lagunas de estabilización». Lima.

Ortega, Andrade. 2006. Phosphorus. Florida: DISSERTATION.

RAITEC. 2009. «Informe del ensayo interlaboratorio para fangos activos». Sevilla.

Ramalho, Rita. 1996. Tratamiento de aguas residuales. Barcelona: Reverte S.A.

Riera, Antony. 2011. «EDAR para una población de más de 100.000 habitantes equivalentes. En A. Garcés Martínez». Barcelona: Tesis de Maestría.

Zornoza, Arcenegui. 2007. EDAR, Análisis rápido macroscópico y microscópico para fangos activos. Un enfoque práctico en el control de EDAR. Valencia. 4-8.

Fecha de recepción: 09 de setiembre de 2016

Fecha de aceptación: 06 de diciembre de 2016

\section{Correspondencia}

Vladimir Alfonso Leon Menacho

vladimirmenacho@gmail.com 